BRAVILLAN JOURNAL

www.bjournal.com.br
ISSN 0100-879X

Volume 44 (7) 606-728 July 2011

BIOMEDICAL SCIENCES

AND

CLINICAL INVESTIGATION

Braz J Med Biol Res, July 2011, Volume 44(7) 688-693

doi: 10.1590/S0100-879X2011007500082

In vivo photorelease of GABA in the mouse cortex

V. Lopes-dos-Santos, J. Campi, O. Filevich, S. Ribeiro and Etchenique

The Brazilian Journal of Medical and Biological Research is partially financed by

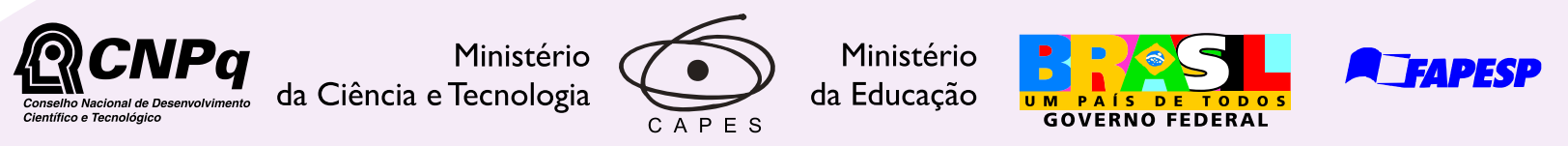

Institutional Sponsors
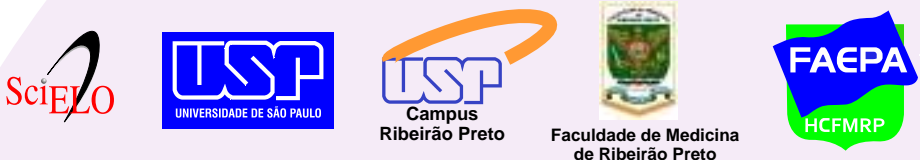

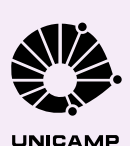

Ф SHIMADZU

GE Healthcare
Hotsite of proteomics metabolomics developped by:

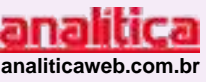




\title{
In vivo photorelease of GABA in the mouse cortex
}

\author{
V. Lopes-dos-Santos ${ }^{1,2}$, J. Campi $^{3}$, O. Filevich ${ }^{3}$, S. Ribeiro ${ }^{1,2}$ \\ and R. Etchenique ${ }^{3}$ \\ ${ }^{1}$ Instituto do Cérebro, Universidade Federal Rio Grande do Norte, Natal, RN, Brasil \\ 2 Instituto Internacional de Neurociências de Natal "Edmond e Lily Safra", Natal, RN, Brasil \\ ${ }^{3}$ Departamento de Química Inorgánica, Analítica y Química Física, INQUIMAE, \\ Facultad de Ciencias Exactas y Naturales, Universidad de Buenos Aires, Buenos Aires, Argentina
}

\begin{abstract}
Electrical stimulation has been used for more than 100 years in neuroscientific and biomedical research as a powerful tool for controlled perturbations of neural activity. Despite quickly driving neuronal activity, this technique presents some important limitations, such as the impossibility to activate or deactivate specific neuronal populations within a single stimulation site. This problem can be avoided by pharmacological methods based on the administration of receptor ligands able to cause specific changes in neuronal activity. However, intracerebral injections of neuroactive molecules inherently confound the dynamics of drug diffusion with receptor activation. Caged compounds have been proposed to circumvent this problem, for spatially and temporally controlled release of molecules. Caged compounds consist of a protecting group and a ligand made inactive by the bond between the two parts. By breaking this bond with light of an appropriate wavelength, the ligand recovers its activity within milliseconds. To test these compounds in vivo, we recorded local field potentials (LFPs) from the cerebral cortex of anesthetized female mice (CF1, 60-70 days, 20-30 g) before and after infusion with caged $y$-amino-butyric-acid (GABA). After 30 min, we irradiated the cortical surface with pulses of blue light in order to photorelease the caged GABA and measure its effect on global brain activity. Laser pulses significantly and consistently decreased LFP power in four different frequency bands with a precision of few milliseconds $(P<0.000001)$; however, the inhibitory effects lasted several minutes $(P<0.0043)$. The technical difficulties and limitations of neurotransmitter photorelease are presented, and perspectives for future in vivo applications of the method are discussed.
\end{abstract}

Key words: Caged compounds; Neural stimulation; GABA; Cerebral cortex; Photorelease

\section{Introduction}

Since Luigi Galvani discovered that static electricity applied to nerves could evoke muscle contractions, bioelectricity has been a major topic in biology and subsequently in neuroscience. Several studies in the past century demonstrated how nerve cells exchange information in the form of ionic currents and biochemical processes $(1,2)$. The search for a better understanding of these phenomena has made extensive use of direct brain stimulation. Electrical microstimulation, for instance, led to fundamental discoveries regarding perception, motor activity, memory consolidation, and the treatment of brain-related illnesses (3-6). In spite of the many successes of electrical stimulation, criticism has been raised due to the simple fact that this kind of electrical current is not a natural phenomenon in the brain. In addition, the spatial resolution is very difficult to control because dendrites, soma and axons are all sensitive to this kind of stimulation, making it impossible to establish precisely which neurons are affected. Zemelman et al. (7), based on the biochemistry of retinal phototransducers, developed a method capable of controlling the firing rates of genetically designated neurons in vitro using white light. Thus, it was possible to target functional populations rather than anatomical locations (8). Despite its many exquisite advantages, such optogenetical methods are still unavailable to most researchers. A simpler and more affordable alternative for the photostimulation of neural circuits is the use of caged compounds. A caged compound is the union of a compound of interest with a chemical cage, which renders the compound ineffectual. The term "caging" was first used by Kaplan et al. (9) with reference to a

Correspondence: R. Etchenique, Departamento de Química Inorgánica, Analítica y Química Física, INQUIMAE, Facultad de Ciencias Exactas y Naturales, Universidad de Buenos Aires, Ciudad Universitaria Pabellón 2, Piso 3, C1428EHA, Buenos Aires, Argentina. E-mail: rober@qi.fcen.uba.ar

Received November 1, 2010. Accepted June 9, 2011. Available online July 8, 2011. Published July 25, 2011. 
protected compound, so called "caged ATP", able to release adenosine 5'-triphosphate upon illumination. By irradiating the complex with light of appropriate wavelength, the compound is irreversibly released from the cage, becoming free to interact with the environment. Thus, the use of caged neurotransmitters may bypass the limitations of the electrical stimulation techniques cited above, making this new kind of stimulation a promising alternative to the use of electrical stimulation.

The first caged agonists for neurotransmitter receptors were activators of acetylcholine receptors released by UV irradiation (10). Some years later the first caged glutamate was developed, and its application to brain slices led to a better understanding of the connection patterns in neurons of the visual cortex (11). Spatial resolution, however, was still a problem due to the scattering properties of UV light. Another solution came from the development of compounds that could be uncaged by visible wavelengths, allowing greater tissue penetration, less light scattering, and better spatiotemporal resolution, while at the same time providing greater simplicity and lower cost (12). Further advantages are presented by the development of caged molecules that could be photolyzed by two-photon irradiation (13).

Caged $y$-amino-butyric-acid (GABA) compounds have also been introduced, being used to suppress experimental seizures in brain slices (14) and to investigate GABA receptors in patch clamp recordings of rat hippocampal slices (15). Visible light-sensitive inorganic cages using a ruthenium complex (ruthenium bipyridines) as a photosensor for visible light $(16,17)$ have been recently developed for both GABA (RuBiGABA) (18) and glutamate (RuBiGlutamate) (16) with no apparent toxicity and with faster dynamics than organic approaches. In the present study, we demonstrate for the first time that the photorelease of RuBiGABA-PMe a modified version of RuBiGABA, with a visible light pulse successfully suppresses cortical local field potential (LFP) activity in anesthetized animals with high temporal resolution. Our experiments, however, did not address caveats related to the spatial resolution of in vivo photostimulation by caged compounds. In this sense, we discuss the technical limitations of the caged compound method and propose new experiments to explore its advantages.

\section{Material and Methods}

\section{Animals and surgical procedures}

Five adult female CF1 mice (FUNDACAL, Buenos Aires, Argentina; age: $60-70$ days, weight: $20-30 \mathrm{~g}$ ) were anesthetized with $100 \mathrm{mg} / \mathrm{kg}$ ketamine and $8.0 \mathrm{mg} / \mathrm{kg}$ xylazine, ip. A craniotomy with a $\sim 3-\mathrm{mm}^{2}$ circular area was drilled in the skull bone over the primary somatosensory cortex of each mouse (stereotaxic coordinates of the craniotomy center: $\mathrm{ML}=1.7 \mathrm{~mm}, \mathrm{AP}=-0.3 \mathrm{~mm}$ ). The experiments were performed in accordance with the National Institutes of Health Guide for the Care and Use of Laboratory Animals
(NIH publication 80-23/96) and Argentinian regulations and were approved by the Ethics Committee of Animal Usage in Research of the Alberto Santos Dumont Research Support Association, Brazil (protocol\#3/2009). All efforts were made to minimize animal suffering and to reduce the number of animals used. Due to physicochemical artifacts, data from 2 animals were not utilized.

\section{RuBiGABA-PMe 3 synthesis}

The precursor complex cis-[Ru(bpy $)_{2}\left(\mathrm{PMe}_{3}\right) \mathrm{Cl}_{3} \mathrm{PF}_{6}$ (bpy $=2,2$ 'bipyridine and $\mathrm{PMe}_{3}=$ trimethylphosphine) was obtained by the following procedure: $520 \mathrm{mg} \mathrm{Ru}(\mathrm{bpy})_{2} \mathrm{Cl}_{2}$ (19) was suspended in $20 \mathrm{~mL}$ of a $1: 1$ mixture of methanol and water, and refluxed under $\mathrm{N}_{2}$. A 1.2-mL aliquot of 1 M trimethylphosphine in tetrahydrofuran (THF; 324108, Aldrich, USA) was added with a syringe. The reaction was monitored by UV-Vis spectroscopy. In some cases, additional phosphine solution was added. Once the UV-Vis spectrum was stable, methanol and excess phosphine were removed by vacuum distillation. The resulting aqueous solution was filtered to remove any solids, and precipitated by the addition of excess potassium hexafluorophosphate $\left(\mathrm{KPF}_{6}\right)$ at $0^{\circ} \mathrm{C}$. The dark orange solid was washed three times with cold water and dried. The yield was $93 \%$. The next part of the synthesis took place under filtered orange light at $\lambda>580 \mathrm{~nm}$.

The photoactive complex, cis-[Ru(bpy $)_{2}\left(\mathrm{PMe}_{3}\right)$ $(\mathrm{GABAH})]\left(\mathrm{PF}_{6}\right)_{2}$ was obtained as follows: $110 \mathrm{mg}$ $\left[\mathrm{Ru}(\mathrm{bpy})_{2}\left(\mathrm{PMe}_{3}\right) \mathrm{Cl}_{\mathrm{PF}} \mathrm{PF}_{6}\right.$ was dissolved in $2 \mathrm{~mL}$ acetone. A suspension of $2 \mathrm{~mL}$ water with $200 \mathrm{mg}$ of a chloridecontaining anionic exchange resin (DOWEX $2 \times 8$ ) was added and stirred for $10 \mathrm{~min}$. The resulting $\left[\mathrm{Ru}(\mathrm{bpy})_{2}\left(\mathrm{PMe}_{3}\right)\right.$ $\mathrm{Cl}] \mathrm{Cl}$ solution was filtered to remove the resin, $500 \mathrm{mg}$ GABA and $1.8 \mathrm{~mL} 1 \mathrm{M} \mathrm{NaOH}$ were then added, and the resulting mixture was heated for $5 \mathrm{~h}$ to $80^{\circ} \mathrm{C}$. One milliliter saturated $\mathrm{KPF}_{6}$ was added and the resulting precipitate was discarded. The solution was then cooled to $0^{\circ} \mathrm{C}$ and acidified by the addition of $5 \mathrm{M} \mathrm{HCl}$ to $\mathrm{pH} \sim 2.0$. The final compound, - $\left[\mathrm{Ru}(\mathrm{bpy})_{2}\left(\mathrm{PMe}_{3}\right)(\mathrm{GABAH})\right]\left(\mathrm{PF}_{6}\right)_{2}$, precipitated upon the addition of excess $\mathrm{KPF}_{6}$. The yellow-orange solid was then washed with cold water and dried. The yield was $46 \%$. Full chemical characterization of the compound obtained will be published elsewhere.

\section{Experimental procedures}

After surgery, each mouse was placed in a dark box and the LFPs were recorded with a sharp microelectrode ( $10 \mathrm{M} \Omega$ at $1 \mathrm{kHz}$; A-M Systems Inc., USA) connected to a Neuroprobe 1600 (A-M Systems Inc.) amplifier and an A/D signal acquisition board (RTX-03B(II), Costronic Co. Ltd., Taiwan) operating at a $250-\mathrm{Hz}$ sampling rate and running in the custom-made QBasic software. Throughout each experiment, images of the mouse brain were continuously recorded by an infrared camera. Laser pulses were delivered by a 4-mW 473-nm wavelength DPSS laser (Shangai 
Laser \& Optics Century Co., China) to release the caged compound in the mouse's brain. Pulse timing was controlled by the computer. A lens fixed to a remote control motor allowed the experimenter to target the light stimulus on the area of interest. The recordings were divided in $\sim 30$-min sessions: i) baseline pre-compound application: saline was applied to the exposed area and 1-s laser pulses were delivered; ii) baseline post-compound application: $50 \mu \mathrm{L}$ $250 \mu \mathrm{M}$ RuBiGABA-PMe ${ }_{3}$ diluted in saline was applied over the exposed brain area using an air displacement pipette of a $100-\mu \mathrm{L}$ volume; iii) post-light stimulus session: a 1-s laser pulse was delivered on the region of interest in order to release the caged GABA.

\section{Signal analysis}

In order to evaluate how compound infusion and lightstimulus photorelease affect LFP power, we first decomposed the signals into 4 non-redundant components using biorthogonal spline wavelets. The resulting components were analyzed in 4 frequency bands of interest: delta $(0.5$ to $3.9 \mathrm{~Hz}$ ), theta (3.9 to $7.8 \mathrm{~Hz}$ ), alpha ( 7.8 to $15.6 \mathrm{~Hz}$ ), and beta $(15.6$ to $31.25 \mathrm{~Hz})$. Since calculation of the root mean square would require binning and therefore would decrease the temporal resolution, we calculated the amplitude envelope of the signals using a Hilbert transform, as shown in Figure 1A. The square of the amplitude envelope signal can be understood to be the instantaneous energy of the signal. Projecting the signals onto continuous Morlet wavelets and again calculating the amplitude envelope using a Hilbert transform, we plotted the wavelet spectrogram of the LFPs.
The use of the continuous wavelet transform improved the temporal resolution in spectrogram calculations, an essential feature for evaluating transitions. After calculation, the data were subjected to non-parametric statistical testing (Kruskal-Wallis test followed by Dunn-Sidák correction for the number of comparisons).

\section{Results}

Figure 1B shows the wavelet spectrogram for mouse 1 , which was the best case. The first vertical line indicates the moment of RuBiGABA-PMe 3 application and the second line indicates the time when the laser stimulus was flashed. Note that neural activity drops abruptly after the light pulse. Changes in the statistical stationarity of the LFP instantaneous amplitude within 4 different frequency bands were calculated at the boundaries of compound application and laser stimulation.

In order to evaluate short-term effects, we compared 1-s windows pre- and post-transition using amplitude envelopes of the signal wavelet filtered for the frequency bands of interest. The group result was calculated by adding up the distributions of three mice after z-score normalization (Figure 2A). LFP power at all frequency bands differed significantly across transitions $(P<0.000001$, Mann-Whitney rank-sum test).

In order to evaluate the long-term effects of compound application and light stimulus in different frequency bands, we applied fast Fourier transform to 10 non-overlapping 2.5min windows before and after each transition. The pairs of
A

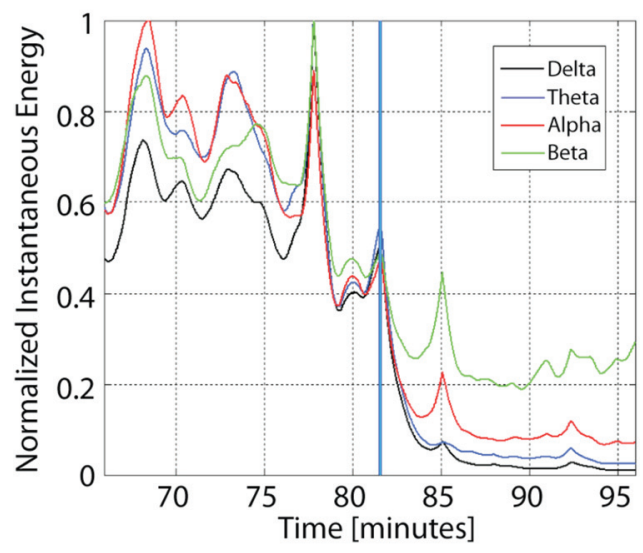

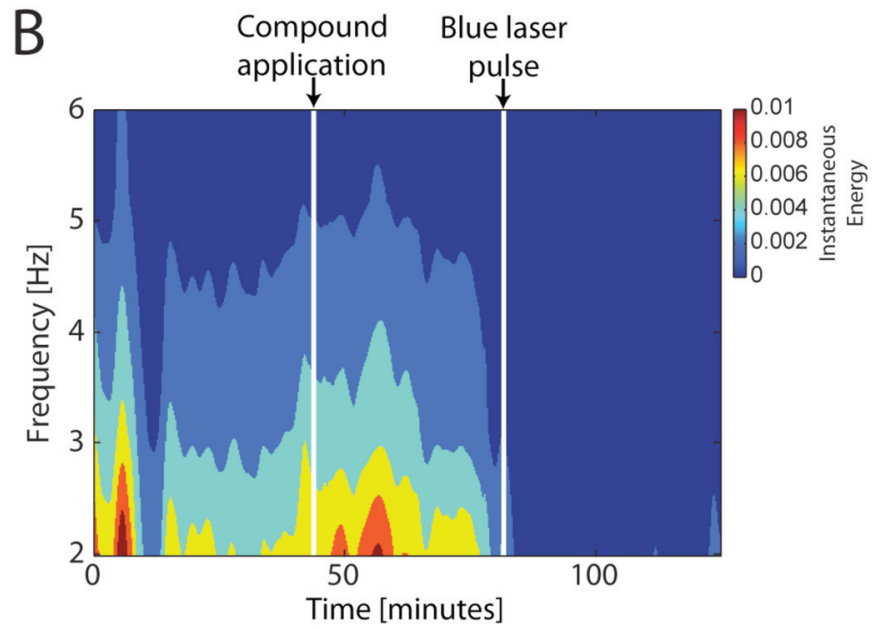

Figure 1. The photorelease of $y$-amino-butyric-acid (GABA) decreases local field potentials (LFP). A, Instantaneous energy calculated by the amplitude envelope of the analytical representation (AR) of the LFP signals for four frequency bands. The AR of a signal was computed using the Hilbert transform, which provides high temporal resolution for analyses at transitions. The blue vertical line indicates the exact time when the laser pulse caused inhibition of neural activity. A clear reduction of all frequency bands was noted following the laser pulse. $B$, A wavelet spectogram for Mouse 1 showing neural inhibition at the time of the laser pulse, which lasted at least $40 \mathrm{~min}$. 


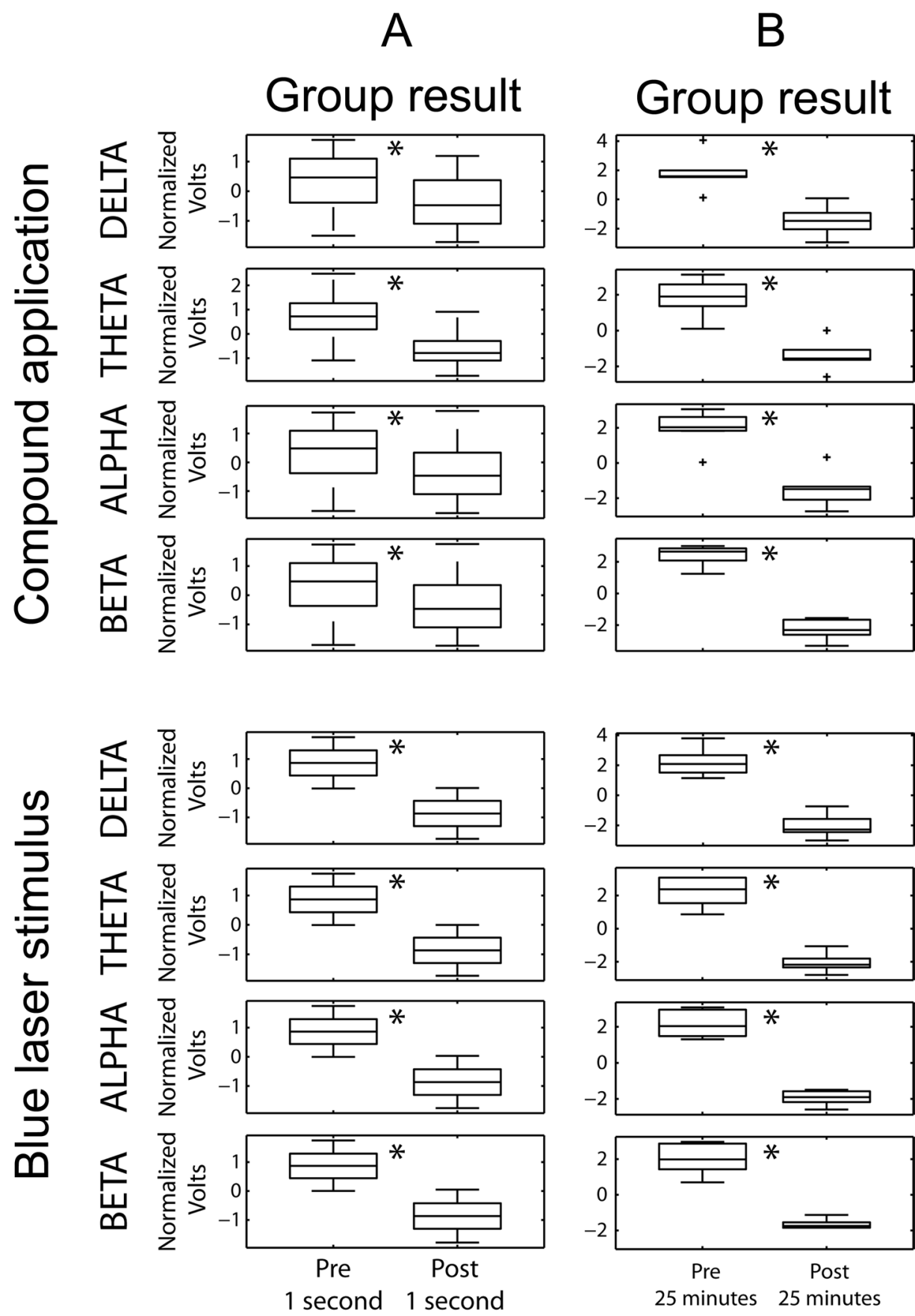

Figure 2. Short- and long-term effects of the infusion and photorelease of caged $y$-amino-butyric-acid (GABA). A, Boxplots evaluating short-term effects of compound application and light stimulus $(\mathrm{N}=$ 3 mice). Calculation of the amplitude envelope in wavelet-filtered bands allows for high temporal resolution. We show that local field potential (LFP) amplitude distribution of windows of $1 \mathrm{~s}$ pre- and post-transitions are statistically different (pre vs post, ${ }^{*} \mathrm{P}<0.000001$, Mann-Whitney-Wilcoxon test). $B$, Boxplots show the long-term changes in the LFP voltage amplitudes in 4 frequency bands for 3 mice at transition times. Each boxplot represents voltage levels within a specific frequency band calculated by fast Fourier transform in ten non-overlapping 2.5-min windows. Thus, 25-min pre- and post-compound application and light stimulus are evaluated. All transitions differed significantly, indicating long-term effects of both compound application and laser pulse stimuli on LFP power $\left({ }^{*} \mathrm{P}<\right.$ 0.0043, Mann-Whitney-Wilcoxon test). 
10 measurements before and after each transition in every frequency band were compared by the Wilcoxon-MannWhiney test. Group results in Figure 2B were calculated as in Figure 2A. For long-term effects, mouse 1 showed a non-significant trend to an increase of the LFP energy after compound application (delta: $P=0.065$; theta: $P=$ 0.065 ; alpha: $P=0.442$, and beta: $P=0.130$ ). Accordingly, a significant decrease of activity was observed following the light stimulus ( $P=0.0001$ for all frequency bands). Mouse 2 presented a decrease in LFP amplitudes for nearly all frequency bands, both at compound application (delta: $P$ $=0.0019$; theta: $P=0.0002$; alpha: $P=0.0002$, and beta: $P=0.0002$ ) and light stimulus (delta: $P=0.0011$; theta: $P$ $=0.0006$; alpha: $P=0.0011$, and beta: $P=0.0019$ ). For mouse 3 , the energy dropped significantly after compound application (delta: $\mathrm{P}=0.0022$; theta: $\mathrm{P}=0.0022$; alpha: $P=0.0022$, and beta: $P=0.0022)$ but not after the light stimulus (delta: $P=0.8182$; theta: $P=0.6991$; alpha: $P$ $=0.6991$, and beta: $P=0.9372$ ), indicating that most of the caged GABA was already released before the laser pulse was applied. Group results presented statistically significant differences for both transitions $(P=0.0022$ for all frequency bands).

\section{Discussion}

In this study, we describe how GABA photorelease with visible light affects different frequency bands of the LFP recorded from the cerebral cortex of anesthetized mice. We show that a blue laser pulse cast over the area infused with RuBiGABA-PMe 3 can significantly inhibit neural activity.

Some technical problems are worth mentioning. First, there is a concern regarding the sensitivity of the caged complex to visible light. Since we have chosen to use very high concentrations in order to attempt complete inhibition of cortical activity, it is very difficult to avoid residual photorelease by environmental light. For this reason it is strongly recommended that the compound be stored as a solid, and be dissolved to proper concentration as close as possible to the application time. It is also important to ensure that the experimental environment is completely dark. Some undesired release of GABA prior to light stimulation can be seen in the data from two mice (as commented above), which show a decrease of LFP power following compound application. On the other hand, in mouse 1 the LFP power slightly increased in all frequency bands after compound application, a result that can be explained by a previously reported GABA antagonism by RuBiGABA (17).

The intrinsic disturbance of neural activity caused by the application of the caged compound, whether from GABAantagonism effects of the caged compound or from the GABA unintended release, is in fact objectionable. It is clear from our results that when the compound application causes a marked drop in neural activity by itself, the subsequent light pulse is less effective. The strongest inhibition elicited by the laser stimulus occurred when application of the compound did not significantly alter LFP energy levels.

These problems can be diminished with further refinement of the method, including the determination of best concentration levels for in vivo applications. In addition, since RuBiGABA has been shown not to be toxic (17), it is in principle possible to inject low concentrations into the blood stream of mice, although we have no clear evidence that RuBiGABA can cross the blood-brain barrier. Another alternative would be to inject the caged compound into the brain ventricles. The most convenient compound concentration and the best duration of the light stimuli will vary according to the experiment. We have used both high concentrations and long stimulus durations in order to release a large quantity of GABA at once.

It must be noted that our results do not show that this kind of neural stimulation can be used in applications that require high spatial resolution. In order to explore the spatiotemporal limitations of our approach, one may need to use single unit recordings in order to evaluate how the observed effects affect individual cells immersed in the cerebral cortex. By using more refined electrophysiological techniques it would be possible to record single neuronal cells spatially distributed in the cortex, and therefore, to evaluate how the compound diffusion and the scattering of laser pulses influence the spatial precision of the stimulation. In addition, since the stimulation effects we observed were in fact long-lasting, we believe that single unit recordings may answer the question of whether the limited release of caged GABA can be used to produce short-term inhibitory effects.

Although the use of caged neurotransmitters for in vivo applications must still overcome some hurdles and be more rigorously tested, our results indicate that neural perturbation by the photorelease of caged compounds may soon be widely used in experimental and medical applications. Since light is employed to affect the release, one can temporally separate the injection of the compound from the photorelease, making it possible to activate the compound when it is well diffused, homogeneously and simultaneously affecting a larger area of the brain, with greater temporal resolution. RuBiGABA-PMe 3 may be used as a substitute for GABA agonists such as muscimol. The use of other neurotransmitters with different functions, such as glutamate or serotonin (20), opens a wide range of possibilities. The possibility in controlling brain activity by laser pulses is potentially a very important application in behavioral studies, since light pulses do not disturb behavior. For instance, this approach could be used to inhibit brain areas during memory retention, or to stimulate the brain during specific wakesleep stages. Although we have not yet mastered the use of caged compounds for applications to behaving animals, this approach can probably be used as a complementary methodology for these kinds of experiments. 


\section{Acknowledgments}

We would like to thank Marcelo Salierno for technical assistance during preliminary experiments. Research sup-

\section{References}

1. Eccles JC. The synapse: from electrical to chemical transmission. Annu Rev Neurosci 1982; 5: 325-339.

2. Hodgkin AL, Huxley AF. A quantitative description of membrane current and its application to conduction and excitation in nerve. J Physiol 1952; 117: 500-544.

3. Bliss TV, Lomo T. Long-lasting potentiation of synaptic transmission in the dentate area of the anaesthetized rabbit following stimulation of the perforant path. J Physiol 1973; 232: 331-356.

4. Graziano MS, Taylor CS, Moore T. Complex movements evoked by microstimulation of precentral cortex. Neuron 2002; 34: 841-851.

5. Stehberg J, Levy D, Zangen A. Impairment of aversive memory reconsolidation by localized intracranial electrical stimulation. Eur J Neurosci 2009; 29: 964-969.

6. Wichmann T, DeLong MR. Deep brain stimulation for neurologic and neuropsychiatric disorders. Neuron 2006; 52: 197-204.

7. Zemelman BV, Lee GA, Ng M, Miesenbock G. Selective photostimulation of genetically chARGed neurons. Neuron 2002; 33: 15-22.

8. Gunaydin LA, Yizhar O, Berndt A, Sohal VS, Deisseroth K, Hegemann P. Ultrafast optogenetic control. Nat Neurosci 2010; 13: 387-392.

9. Kaplan JH, Forbush B III, Hoffman JF. Rapid photolytic release of adenosine 5'-triphosphate from a protected analogue: utilization by the Na:K pump of human red blood cell ghosts. Biochemistry 1978; 17: 1929-1935.

10. Walker JW, McCray JA, Hess GP. Photolabile protecting groups for an acetylcholine receptor ligand. Synthesis and photochemistry of a new class of o-nitrobenzyl derivatives and their effects on receptor function. Biochemistry 1986; 25: 1799-1805. ported by a CNPq fellowship, and by grants from FINEP, CAPES Secyt, CONICET, and AASDAP. R. Etchenique is a member of CONICET.

11. Dalva MB, Katz LC. Rearrangements of synaptic connections in visual cortex revealed by laser photostimulation. Science 1994; 265: 255-258.

12. Shembekar VR, Chen Y, Carpenter BK, Hess GP. A protecting group for carboxylic acids that can be photolyzed by visible light. Biochemistry 2005; 44: 7107-7114.

13. Kramer RH, Fortin DL, Trauner D. New photochemical tools for controlling neuronal activity. Curr Opin Neurobiol 2009; 19: $544-552$.

14. Yang XF, Schmidt BF, Rode DL, Rothman SM. Optical suppression of experimental seizures in rat brain slices. Epilepsia 2010; 51: 127-135.

15. Molnar P, Nadler JV. Gamma-aminobutyrate, alpha-carboxy2-nitrobenzyl ester selectively blocks inhibitory synaptic transmission in rat dentate gyrus. Eur J Pharmacol 2000; 391: 255-262.

16. Salierno M, Marceca E, Peterka DS, Yuste R, Etchenique R. A fast ruthenium polypyridine cage complex photoreleases glutamate with visible or IR light in one and two photon regimes. J Inorg Biochem 2010; 104: 418-422.

17. Rial Verde EM, Zayat L, Etchenique R, Yuste R. Photorelease of GABA with visible light using an inorganic caging group. Front Neural Circuits 2008; 2: 2.

18. Zayat L, Noval MG, Campi J, Calero Cl, Calvo DJ, Etchenique R. A new inorganic photolabile protecting group for highly efficient visible light GABA uncaging. Chembiochem 2007; 8: 2035-2038.

19. Viala $\mathrm{C}$, Coudret $\mathrm{C}$. An expeditious route to cis-Ru(bpy)(2) $\mathrm{Cl}-2$ (bpy=2,2'-bipyridine) using carbohydrates as reducers. Inorganica Chim Acta 2006; 359: 984-989.

20. Zayat L, Salierno M, Etchenique R. Ruthenium(II) bipyridyl complexes as photolabile caging groups for amines. Inorg Chem 2006; 45: 1728-1731. 